\title{
A General Model for EV Drivers' Charging Behavior
}

\author{
Zohreh Fotouhi, Massoud Reza Hashemi, Hamed Narimani, and I. Safak Bayram
}

\begin{abstract}
The increasing adoption of electric vehicles (EVs) due to technical advancements and environmental concerns requires wide deployment of public charging stations (CSs). In order to accelerate the $\mathrm{EV}$ penetration and predict the future CSs requirements and adopting proper policies for their deployment, studying the charging behavior of $\mathrm{EV}$ drivers is inevitable. This paper introduces a stochastic model that takes into the consideration the behavioral characteristics of $\mathrm{EV}$ drivers in particular in terms of their reaction to the EV battery charge level when deciding to charge or disconnect at a CS. The proposed model is applied in two case studies to describe the resultant collective behavior of $\mathrm{EV}$ drivers in a community using real field EV data obtained from a major North American campus network and part of London urban area. The model fits well to the datasets by tuning the model parameters. The sensitivity analysis of the model indicates that changes in the behavioral parameters affect the statistical characteristics of charging duration, vehicle connection time and $\mathrm{EV}$ demand profile, which has a substantial effect on congestion status in CSs. This proposed model is then applied in several scenarios to simulate the congestion status in public parking lots and predict the future charging points needed to guarantee the appropriate level of service quality. The results show that studying and controlling the EV drivers' behavior leads to a significant saving in CS capacity and results in consumer satisfaction, thus, profitability of the station owners.
\end{abstract}

Index Terms- Electric Vehicle (EV), characteristic modeling, congestion control, electric vehicle charging.

\begin{tabular}{ll} 
& \multicolumn{1}{c}{ NomenCLATURE } \\
$C P$ & Charging Point. \\
$C S$ & Charging Station. \\
$M C S$ & Monte Carlo Simulation. \\
$M D P$ & Markov Decision Process. \\
$c_{i}$ & The state of the EV connection to the CP in \\
& Markov chain with i\% level of charge. \\
$d_{i}$ & The state of the EV disconnection from the CP \\
& in Markov chain with i\% level of charge. \\
$p_{i}$ & The probability of EV driver's decision to dis- \\
& connect with i\% EV's level of charge, defined \\
& by logistic function. \\
$q_{i}$ & The probability of EV driver's decision to \\
& connect while the EV is moving with i\% EV's \\
& level of charge, defined by logistic function. \\
$\epsilon_{t}$ & The probability of EV parking in time t when \\
& the EV is disconnected from CP.
\end{tabular}

Copyright (c) 2015 IEEE. Personal use of this material is permitted. However, permission to use this material for any other purposes must be obtained from the IEEE by sending a request to pubs-permissions@ieee.org.

$Z$.Fotouhi, M. Hashemi and H. Narimani are with the Department of Electrical and Computer Engineering, Isfahan University of Technology, Isfahan 84156-83111, Iran (e-mail: z.fotouhi@ec.iut.ac.ir, h.narimani@cc.iut.ac.ir, hashemim@cc.iut.ac.ir).

I. S. Bayram (corresponding author) is with Qatar Environment and Energy Research Institute, Hamad Bin Khalifa University, Doha, Qatar (e-mail: ibayram@hbku.edu.qa).

\begin{tabular}{|c|c|}
\hline$x_{p}$ & $\begin{array}{l}\text { The central point of the gradient in logistic } \\
\text { function of } p_{i} \text {, as a basis for the driver's } \\
\text { decision to disconnect. }\end{array}$ \\
\hline$x_{q}$ & $\begin{array}{l}\text { The central point of the gradient in logistic } \\
\text { function of } q_{i} \text {, as a basis for the driver's de- } \\
\text { cision to connect. }\end{array}$ \\
\hline$k_{p}$ & $\begin{array}{l}\text { The coefficient of gradient change from zero } \\
\text { to one in logistic function of } p_{i} \text {. }\end{array}$ \\
\hline$k_{q}$ & $\begin{array}{l}\text { The coefficient of gradient change from one to } \\
\text { zero in logistic function of } q_{i} \text {. }\end{array}$ \\
\hline$S O C_{\min }$ & $\begin{array}{l}\text { The minimum threshold of battery state of } \\
\text { charge to connect. }\end{array}$ \\
\hline$S O C_{\max }$ & $\begin{array}{l}\text { The maximum threshold of battery state of } \\
\text { charge to disconnect. }\end{array}$ \\
\hline$P_{s}\left(c_{i}\right)$ & The probability of state $c_{i}$ \\
\hline$P_{s}\left(d_{i}\right)$ & The probability of state $d_{i}$. \\
\hline $\operatorname{Pr}\{k=a\}$ & $\begin{array}{l}\text { The probability of being in a numbers of se- } \\
\text { quential connection states from } c_{j} \text { to } c_{(j+a)} \text {, } \\
\text { for every possible initial state } c_{j} \text {. }\end{array}$ \\
\hline$P_{\text {block }}$ & The blocking probability of completely dis- \\
\hline
\end{tabular}

\section{INTRODUCTION}

The use of battery electric vehicles (BEVs) and plug-in hybrid electric vehicles (PHEVs) in the transportation networks leads to a significant reduction in the use of fossil fuels consumption with direct effect on air pollution. As a result, accelerating the $\mathrm{EV}$ deployment rate is one of the main objectives of governments policies on air quality and decarbonisation. The continuous development of EV powertrain, battery and charge technologies and energy management strategies have further increased the EV market penetration in recent years [1], [2]. Hopefully, the growing adoption of EVs will improve the worldwide transportation sustainability [3]. Therefore, exploring the facilitators and barriers to the diffusion of EVs is quite important [4]. The Energy Outlook report by IEA stipulates that the number of EVs on the roads would increase from 2 to 160 million by 2030 worldwide [5].

The utilization of EVs is not without its advantages and disadvantages concerning its operation and planning of power systems. The negative impacts resulting from the electrification of transportation network and their influence on generation, transmission, and distribution of power grids motivate the researchers to pursue studies regarding technical, operational and economic aspects of this issue [6], [7]. Facilitating the rapid growth of EVs and enjoying its benefits requires the design and development of private and publicly accessible charging stations (CSs) [8]. In this regard, appropriate modeling of charging behavior of EV drivers is our main focus which is 
essential in forecasting the CSs requirements and adopting proper policies for their development, when the number of EVs will increase in the future.

Various studies have been performed to model the mobility behavior of EVs using data analysis [9]-[11] and/or mathematical methods [12]-[19]. A data mining model is introduced in [11] to investigate the characteristics of EV charging load in a geographical area. In [12], uncontrolled EV drivers' behavior during charging is described with Markov chain model to assess the network line loading and the losses due to the increased number of EVs. A Markov model is proposed in [13] to simulate the PHEVs travel behavior by considering the typeof-trip and recharging price flexibility, as well as the choice of switching the fuel type instead of a fast charging option. In this method, the estimation of the transition state probabilities can be very time consuming for all time slots on weekdays due to limited data and particularly considering the need for a high temporal resolution. An agent-based modeling approach is proposed in [15] for predicting the electricity demand for EV charging by considering the influence of the human aggregate behavior on load demand.

In [12] to [15], the collective behavior of the EV fleet drivers is modeled, while [16] to [18] have assumed that the random behavior of a single vehicle is different from the behavior of the population it belongs to. Therefore, they modeled the random behavior of a single vehicle's driver to examine the load profile with more details. A heterogeneous Markov model is presented in [16] to describe the mobility behavior of a single EV and this model is assessed based on a 5 months-long data gathered in Denmark. This data set contains information on whether the vehicle was driven or not at any time for the same period. The number of parameters in this model is reduced by applying B-Spline functions to a generalized linear model. However, the model proposed in [16] lacks the data regarding any specific assumption about the vehicle's characteristics. A Markov chain model is introduced in [18] to describe the EV driver's behavior with the objective of evaluating his/her reaction when the EV is out of power. The general assumption in [18] is that the EV always leaves home at the beginning of the day with its fully charged battery, charged by a home charger and returns home at the end of the day. The Markov model is adopted to assess the probability of the EV battery getting depleted before returning home in a case study. A probabilistic model for nodal EV charging demand is developed in [19] based on spatial-temporal EV dynamics by using Monte Carlo Simulation (MCS). In this study, random trip chain and Markov Decision Process (MDP) are applied to model individual EV drivers' driving and charging behavior.

In all the previously mentioned papers, the proposed EV mobility models have been utilized in order to describe the statistical distribution of behavioral-related parameters of EV drivers, such as load profile, starting and finishing time of EV trip and EV connection and charging times. Most of these models have been presented based on the driving patterns of internal combustion engine vehicles (ICEVs) without considering the EV drivers' charging habits. The critical issue is that these models cannot be generalized for systems with different EV specifications and increased EV population in the future, especially when CSs could not serve all the EVs because of congestion.

In some studies of EV charging navigation, the EV connection and charging times are analyzed based on real-time power grid and traffic data without developing any behavioral model to describe the EV drivers' charging habits [20], [21]. In [20], the EV terminal estimates the driving, waiting and charging times for different CSs in order to guide the EV driver to be navigated to the proper CS. The charging navigation framework of EVs to fast CSs is formulated by a hierarchal game approach in [21].

On the other hand, there exists many studies in the related literature concerning various aspects of design, development and deployment of CSs, focusing on issues like scheduling the charging of EVs [22], [23], congestion management and resource allocation at CSs [24]-[27], determining the appropriate sites [27]-[29], the role of urban planning [4] and economic aspects of investment planning [30], [31]. However, the impact of the random characteristics of EV drivers' behavior on the distribution characteristics of the behavioral-related parameters in the models have not been addressed explicitly rather some simplifying assumptions have been made. For example, the EV arrivals to a CS are typically modeled as a fixed rate Poisson process, while the charging and parking times are characterized as exponential or Gaussian distributions [19], [25]-[28], [32]-[34]. Such simplifying assumptions about these statistics cannot be generalized to common case. In fact, the different behavioral distributions in the studied models in the literature are because of the different behaviors of the EV drivers in different communities. Furthermore, the EV drivers' behavior affects many aspects of the design challenges of CSs including resource provisioning and investment planning, especially when the EV population grows in the future. To the best of the authors' knowledge, the impact of the EV drivers' behavior on these two critical components have not been addressed in the literature.

To address the above problems, in this paper we use a stochastic model for the EV driver's behavior based on EV battery specifications, the initial battery charge level and the driver's habits. Accordingly, the parameters of this model depend on the behavioral characteristics of a typical driver from the community. The proposed model is assessed through mathematical analysis, case study simulations and comparing the results with the real field data obtained at a major North American university campus network [35] and part of London urban area [36]. By adjusting the parameters, the simulation results taken from the model match the real field data. Furthermore, we show that our model can be adjusted to cover different EV driver's behaviors. We assume that most of the EV drivers have a similar charging behavior in a way that the effect of the minority on the overall system behavior can be ignored. Based on this assumption, we estimate the parameters of a typical driver using the real field data. Subsequently, we run the model by applying these parameters to assess the CS congestion status. Specifically, this method can be utilized for CS development and calculating the number of charging points (CPs) that can provide a certain level of service quality, when the number of EVs grows in the future. The customer blocking 


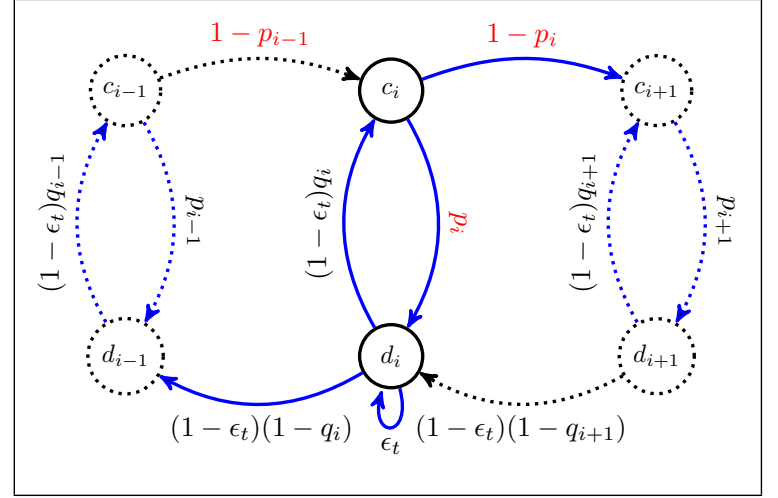

Fig. 1: The proposed stochastic model describing the EV driver's charging behavior

probability can be a good measure of the service quality.

Compared to the existing literature, this is the first study that considers the drivers' behavioral characteristics regarding the charging of the EVs. Furthermore, our proposed behavioral model provides a method for predicting the required power capacity of the CPs with respect to the increase of EVs in the future to prevent congestion in CPs. Therefore, its purpose and practical application would be different. Such a study can help the station owners to develop the CSs in a profitable manner and estimate the investment costs.

The main contributions and results of this work are:

- Proposing a new model to describe a typical EV driver's charging behavior in a given community at CPs, where the model parameters depend on the behavioral characteristics of the vehicle owner, the vehicle specifications and the EV battery level of charge.

- Applying the proposed model to predict the congestion status of CSs by increasing the number of EVs, and to predict future station requirements to achieve the appropriate level of service quality.

- Adjustment and sensitivity analysis of the model's parameters and assessing the system performance by fitting the simulation outputs to real field data.

- Reducing the required number of CPs for a certain level of service quality by encouraging the drivers to modify their EV charging behavior via dynamic pricing.

The remainder of this paper is organized as follows: The system components and the proposed model to describe EV's charging behavior are presented in Section II. The results of the model simulation and its comparison with actual data are provided in Section III. In Section IV, a sensitivity analysis is performed to assess the influence of the new model parameters on the charging behavioral-related parameters of EV drivers. The influence of the model parameters on occurrence of congestion at the CPs and their impact on the future CS requirements are discussed and analyzed in Section V. In Section VI, we have a discussion on the results and Section VII concludes the paper.

\section{STOCHASTIC MODEL FOR EV DRIVERS' CHARGING BEHAVIOR}

In order to model the charging behavior of an $\mathrm{EV}$ driver as a statistical sample of a community, we consider the behavioral reaction of the driver to the battery level of charge. The EV driver usually decides when to connect/disconnect the EV to/from charger based on the current battery level of charge, which is monitored by the dashboard screen. Battery level of charge is an indicator of the available charge stored in the battery. Accurate measurement of this level is necessary for charge/discharge safety and battery health [37]. When the EV battery level of charge drops below a minimum threshold, the driver tends to connect the EV to the charger with a high probability. This minimum threshold is described as $S o C_{\min }$. Similarly, if the EV is connected to CP and the battery level of charge goes beyond a maximum threshold, named $S o C_{\max }$, driver decides to disconnect the EV with a high probability. $S o C_{\min }$ and $S o C_{\max }$ depend on the behavioral characteristics of the driver and his/her driving habits. Moreover, a driver with a higher level of range anxiety tends to connect (resp. disconnect) the EV almost exactly when the battery level of charge reaches to $S o C_{\min }$ (resp. $S o C_{\max }$ ) while others may ignore to connect (resp. disconnect) the EV at right time. Based on these descriptions, we will introduce our proposed model and its parameters in such a way to describe the charging behavior of an EV drivers' community properly.

Now, we explain the details of the proposed stochastic model as follows. Every EV driver can be either in charging, moving or parking situations. When the EV is connected to the $\mathrm{CP}$, the driver decides to remain connected depending on the current energy level of the EV battery. When the EV is disconnected, the driver continues driving towards his destination. The time spent at any $\mathrm{CP}$ is generally random but it also depends on the charge level. Assume that the EV dashboard screen displays the battery energy at $N$ levels. In this case, the amount of remaining energy in the battery can be quantized into integer numbers, that is, $i=0,1,2, \ldots, N$. The level $i=0$ indicates an empty battery and the level $i=N$ indicates a fully charged battery. Based on these assumptions, any EV driver's behavior in choosing either of the above mentioned situations is modeled as a non-homogeneous Markov chain in terms of the energy level of the EV battery. The proposed model has $2(N+1)$ states: $N+1$ states correspond to the charge level while the $\mathrm{EV}$ is connected to the $\mathrm{CP}$ and is receiving power from the grid and the other $N+1$ states correspond to the charging level when the EV is on the road, or stopped but is not connected to the CP. State transition diagram for the proposed model is presented in Fig. 1. Accordingly, the proposed nonhomogeneous Markov chain is defined with a discrete state space $S$ and transition probability matrix $P(t)$, as:

$$
\begin{aligned}
& S=\left\{s_{i}\right\}_{i=0}^{2 N+1}=\left\{c_{i}\right\}_{i=0}^{N} \cup\left\{d_{i}\right\}_{i=0}^{N}, \\
& P(t)=\left[p_{s_{i} s_{j}}(t)\right] \quad s_{i}, s_{j} \in S .
\end{aligned}
$$

$p_{s_{i} s_{j}}(t)$ is the conditional probability of transit from state $s_{i}$ to state $s_{j}$ at time t. The row sum of the transition probability matrix is equal to 1 . The transition probabilities of the proposed model have been defined based on the behavioral parameters $p_{i}, q_{i}$ and $\epsilon_{t}$.

The transition between states will happen as shown in Fig 1. When $\mathrm{EV}$ is connected to the $\mathrm{CP}$ in the state $c_{i}$, the driver 

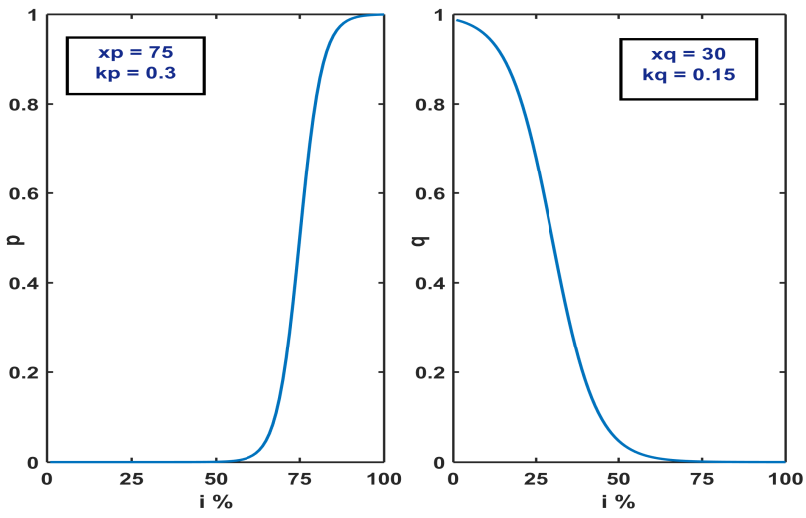

Fig. 2: Logistic functions $p_{i}$ and $q_{i}$ Parameter values : $x_{p}=75$ , $k_{p}=0.3, x_{q}=30, k_{q}=0.15$.

decides to continue charging and increasing the battery energy level with the probability of $1-p_{i}$. After being in this state for a specific duration of time, the EV dashboard screen displays an increment in the battery level of charge. Therefore, EV leaves the state $c_{i}$ towards $c_{i+1}$. Hence, $p_{i}$ is the probability of $\mathrm{EV}$ disconnecting from the $\mathrm{CP}$ and leaving the state $c_{i}$ towards $d_{i}$. When the EV is disconnected from the CP in state $d_{i}$, the driver decides to park (resp. drive) the EV with the probability of $\epsilon_{t}$ (resp. $\left.\left(1-\epsilon_{t}\right)\left(1-q_{i}\right)\right)$. Accordingly, a transition from $d_{i}$ towards $d_{i-1}$ results in decreasing in the level of charge. The probability of connecting the EV to the charger and transition from the state $d_{i}$ towards $c_{i}$ is $\left(1-\epsilon_{t}\right) q_{i}$. Therefore, $q_{i}$ is the probability of connecting to $\mathrm{CP}$ while the $\mathrm{EV}$ is on the road. The battery level of charge in $c_{i}$ and $d_{i}$ states are equal to $i$.

\section{A. Model Parameters}

In order to match the model to actual EV mobility, the $p_{i}$ and $q_{i}$ transition probabilities must be properly defined. $p_{i}$ and $q_{i}$ are subject to the amount of the battery charge level in $c_{i}$ and $d_{i}$, respectively. The logistic functions are applied here to properly define the transition probabilities $p_{i}$ and $q_{i}$, as:

$$
p_{i}=\frac{1}{1+e^{-k_{p}\left(i-x_{p}\right)}} \quad \text { and } \quad q_{i}=\frac{1}{1+e^{-k_{q}\left(-i+x_{q}\right)}}
$$

where $x_{p}, x_{q}, k_{p}$, and $k_{q}$ can be used to define and adjust the driver's behavioral-related parameters. The value of $x_{p}$ (resp. $x_{q}$ ) depends on $S o C_{\max }$ (resp. $S o C_{\min }$ ) and the value of $k_{p}$ (resp. $k_{q}$ ) depends on the level of range anxiety of the driver in deciding about disconnecting (resp. connecting). For example, a driver who is subject to a high level of range anxiety, will be described with a larger $x_{p}$ and/or $x_{q}$ and a larger $k_{p} / k_{q}$. Moreover, the parameter $\epsilon_{t}$ must be set appropriately at all times, in order to determine the driver's tendency to park his/her vehicle for any purpose anywhere without connecting to a CP. For example, if the CPs are located at a university campus, vehicle drivers may have more willingness to connect their vehicle to CPs at class time or lunch hours, while they leave the campus at the end of the day.

The model parameters are defined in a proper manner to characterize a typical driver's charging behavior. This kind of definition has been used in literature. In [21] and [38], the EV charging probability has been described by an exponential function dependent on the battery SoC. Fig. 2 shows $p_{i}$ and $q_{i}$ as the functions of the battery level of charge for some values of system parameters to describe such a driver. Every EV drivers' charging decision depends on the current buttery level of charge. When the driver decides to connect the EV to the CP in a given level of charge with high probability, the probability of disconnecting in the same level of charge will be very low and ignorable, and vice versa. Therefore, the upper levels (resp. lower levels) of $p_{i}$ and $q_{i}$ do not overlap. Although there may always be some hasty people who decide to recharge the EV battery more frequently or disconnect the EV when the level of charge is not high enough, but as mentioned before, we only model the behavior of the common case drivers in the community with common driving and charging habits. In the next section, we will analyze the proposed model mathematically to assess the effect of these parameters on the state probabilities, which indicates the strength of the proposed model in describing the statistics of EV behavioral-related parameters in a CS environment.

\section{B. Mathematical analysis}

In this subsection, we formulate the state probabilities and the probability mass function (pmf) of vehicles' connection time. Let $P_{s}\left(c_{k}\right)$ and $P_{s}\left(d_{k}\right)$ represent the probability of states $c_{k}$ and $d_{k}$ respectively, i.e.,

$$
\begin{aligned}
& P_{s}\left(c_{k}\right) \triangleq \operatorname{Pr}\left\{s=c_{k}\right\} \\
& P_{s}\left(d_{k}\right) \triangleq \operatorname{Pr}\left\{s=d_{k}\right\} .
\end{aligned}
$$

The state probabilities based on the Bayes' rule and the transition probabilities of the model (i.e., $p_{i}, q_{i}$ and $\epsilon_{t}$ ), can be written as,

$P_{s}\left(c_{k}\right)=\left\{\begin{array}{rr}\left(1-p_{k-1}\right) P_{s}\left(c_{k-1}\right)+\left(1-\epsilon_{t}\right) q_{k} P_{s}\left(d_{k}\right) \\ \left(1-\epsilon_{t}\right) P_{s}\left(d_{0}\right) & \text { for } 1 \leq k \leq N \\ \text { for } k=0\end{array}\right.$

$$
\begin{gathered}
P_{s}\left(d_{k}\right)=\left\{\begin{array}{r}
P_{s}\left(c_{k}\right) p_{k}+P_{s}\left(d_{k+1}\right)\left(1-\epsilon_{t}\right)\left(1-q_{k+1}\right) \\
+P_{s}\left(d_{k}\right) \epsilon_{t} \\
\text { for } \quad 0 \leq k \leq N-1, \\
P_{s}\left(c_{N}\right) p_{N}+P_{s}\left(d_{N}\right) \epsilon_{t} \quad \text { for } \quad k=N
\end{array}\right. \\
\text { and } \quad \sum_{k=1}^{N}\left\{P_{s}\left(c_{k}\right)+P_{s}\left(d_{k}\right)\right\}=1 .
\end{gathered}
$$

Note that we have: $q_{0}=1, p_{0}=0$ and $p_{N}=1$. According to the above equations, each one of the state probabilities $P_{s}\left(d_{k}\right)$ can be calculated based on the probabilities of the previous states, $d_{k-1}, d_{k-2}, \ldots, d_{0}$, that is, 


$$
\begin{aligned}
P_{s}\left(d_{1}\right)= & \frac{1-p_{0}}{1-q_{1}} P_{s}\left(d_{0}\right) \\
P_{s}\left(d_{2}\right)= & \frac{-p_{1}\left(1-p_{0}\right)}{1-q_{2}} P_{s}\left(d_{0}\right)+\frac{1-p_{1} q_{1}}{1-q_{2}} P_{s}\left(d_{1}\right) \\
\vdots & \\
P_{s}\left(d_{k}\right)= & \frac{-p_{k-1}}{1-q_{k}} \sum_{i=0}^{k-2}\left\{\prod_{j=i}^{k-2}\left(1-p_{j}\right) q_{i} P_{s}\left(d_{i}\right)\right\} \\
& +\frac{1-p_{k-1} q_{k-1}}{1-q_{k}} P_{s}\left(d_{k-1}\right),
\end{aligned}
$$$$
\text { for } \quad 2<k \leq N
$$

In a similar manner, $P_{s}\left(c_{k}\right)$ is calculated for each state $c_{k}$ :

$$
\begin{aligned}
& P_{s}\left(c_{k}\right)=\left(1-\epsilon_{t}\right) \sum_{i=0}^{k-1}\left\{\prod_{j=i}^{k-1}\left(1-p_{j}\right) q_{i} P_{s}\left(d_{i}\right)\right\} \\
& \quad \text { for } \quad 1 \leq k \leq N
\end{aligned}
$$

Moreover, the probability of being in $a$ sequential connection states is calculated for $a \in\{1,2, \ldots, N\}$ as:

$$
\operatorname{Pr}\{k=a\}=\sum_{j=0}^{N-a}\left\{P_{s}\left(d_{j}\right) q_{j}\left(1-\epsilon_{t}\right) p_{j+a} \prod_{\substack{m=j \\ \text { for } \quad 1 \leq a \leq N}}^{j+a-1}\left(1-p_{m}\right)\right\},
$$

where $\operatorname{Pr}\{k=a\}$ is the probability of being in $a$ sequential connection states from $c_{j}$ to $c_{j+a}$ for some $j$. The probabilities $P_{s}\left(c_{i}\right), P_{s}\left(d_{i}\right)$ and $\operatorname{Pr}\{k=a\}$ are solved numerically and depicted in Fig. 3 for $\epsilon_{t}=0.4$ and $p_{i}$ and $q_{i}$ as shown in Fig. 2.

The effect of different sets of model parameters on $P_{s}\left(c_{i}\right)$ and $P_{s}\left(d_{i}\right)$ have been assessed and the results are depicted in Fig. 3d: The amounts of $e_{1}$ (resp. $\left.e_{2}\right)$ in $P_{s}\left(c_{i}\right)$ and $P_{s}\left(d_{i}\right)$ depend on $x_{q}$ (resp. $x_{p}$ ). $k_{q}$ (resp. $k_{p}$ ) affects on the increasing (resp. decreasing) gradients of these functions. Moreover, a decrease in $\epsilon_{t}$ leads to increasing (resp. decreasing) the maximum probability value in $P_{s}\left(c_{i}\right)$ (resp. $P_{s}\left(d_{i}\right)$ ) (i.e., m). Therefore, the EV connection and disconnection times can be controlled by changing the model parameters. On the other hand, it is observed that the shape of $\operatorname{Pr}\{k=a\}$ changes with parameters $p_{i}$ and $q_{i}$. By considering the constant amount of charge speed, $\operatorname{Pr}\{k=a\}$ describes the duration of being in the connection states. Therefore, the effect of parameters $p_{i}$ and $q_{i}$ on this function is equivalent to their effect on the pmf of charging time, which will be studied in Section IV. As a result, it is expected that the proposed model describes different EV drivers' behaviors in different places by adjusting the model parameters appropriately, which has a direct effect on the statistics of behavioral-related parameters of the system. Therefore, a case study will be assessed in Section III to ensure this ability.

\section{CAse STUdy}

In this section, we validate the proposed stochastic model by adjusting the model parameters in two separate case studies
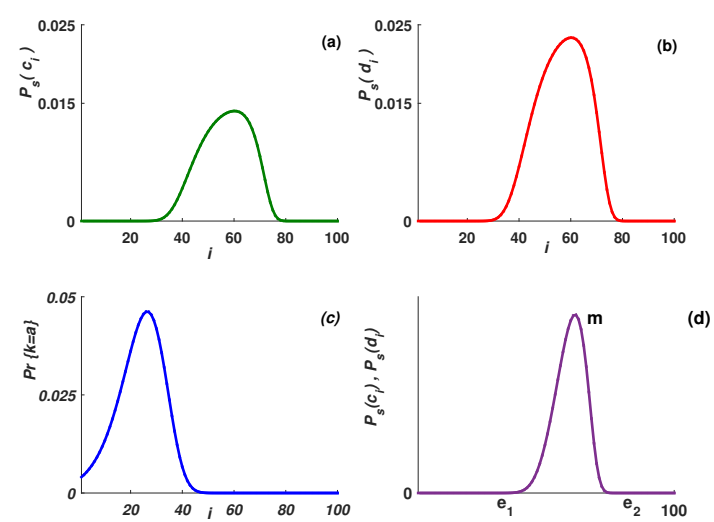

Fig. 3: (a) $P_{s}\left(c_{i}\right)$, (b) $P_{s}\left(d_{i}\right)$, (c) $\operatorname{pr}\{k=a\}$ in terms of the state number, for $\epsilon_{t}=0.4$ and $p_{i}$ and $q_{i}$ shown in Fig. 2

(d) a typical $P_{s}\left(c_{i}\right)$ or $P_{s}\left(d_{i}\right)$

and fitting the model to real data. In these two case studies, the data obtained from the public CPs located at a university campus and part of London urban area are considered, receptively. In both cases, the distance between the CPs is short enough that any EV will have access to a CP if it needs to be charged. There will be no queuing service at the CPs because of long lasting charging periods. If all the chargers are occupied, the driver decides to drive to nearest charger. It is assumed that each CP delivers a constant amount of power during the charging, which is common in EV modeling [39][41]. Also when driving, the EV level of charge decreases with a certain discharging rate, which is determined by the energy consumption coefficient and the average driving velocity of vehicles [13], [41]. Since the data sets are gathered from urban areas, the average driving velocity is considered $44 \mathrm{~km} / \mathrm{s}$ in calculating the EVs' discharging rate. The type of EVs are assumed to be personal vehicles with similar characteristics as Nissan Leaf [42].

TABLE I: Simulation parameters [42]-[44]

\begin{tabular}{lcc}
\hline \multicolumn{1}{c}{ Parameters } & Case 1 & Case 2 \\
\hline Battery capacity $C,(\mathrm{kWh})$ & 30 & 24 \\
$\begin{array}{l}\text { Energy consumption coefficient } \\
E_{c}(k W h / k m)\end{array}$ & 0.186 & 0.155 \\
Average speed $\bar{v}(k m / s)$ & 44 & 44 \\
Battery efficiency $\left(\eta_{c}\right)$ & 0.97 & 0.93 \\
Charging rate $P_{c}(k W)$ & 6.5 & $2.35 / 3.7$ \\
Type of CP & SAE J1772 & IEC62196 \\
On board charger output $P_{o}(k W)$ & 3.6 & 3.3 \\
Full charge time $(h)$ & 8.59 & $11 / 7.8$ \\
Full discharge time $(h)$ & 3.6 & 3.5 \\
\hline
\end{tabular}

The presented model is used to simulate EV drivers' charging behavior in two cases based on real charging events. The simulation procedure is presented in Fig. 4. The key parameters and charging characteristics of the EVs and CPs in these cases are provided in Table I. The number of states 


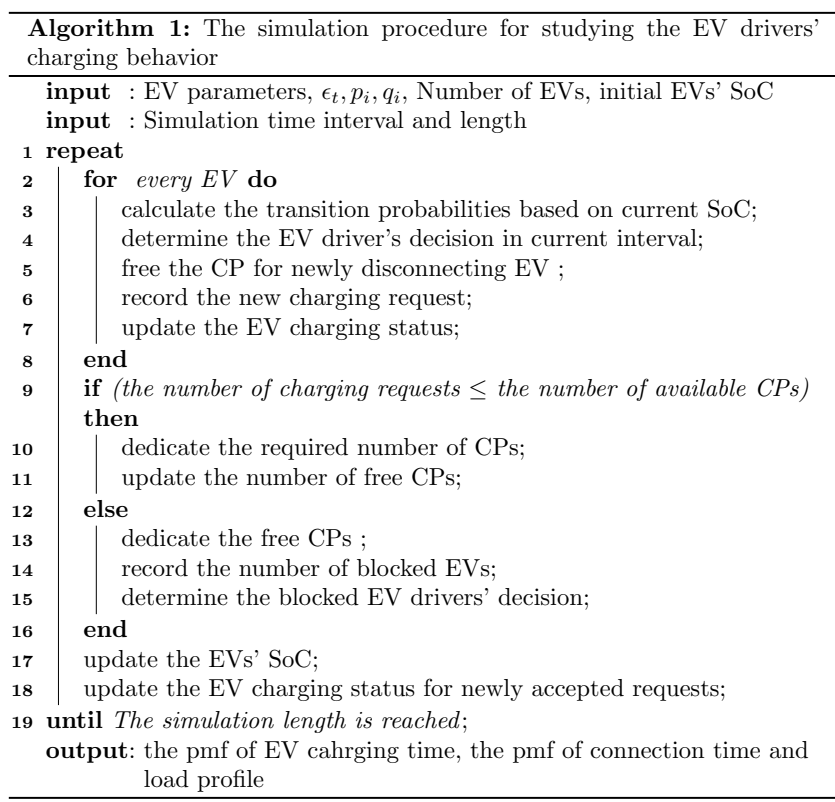

Fig. 4: The simulation procedure using the proposed model

in proposed model is set to $N=100$. The study in Case 1 is described based on the data set obtained at a major North American university campus network [35]. This local EV charging station consists of 11 CPs for public and 6 CPs for fleets all physically located in vehicle parking lots, equipped with AC-level 2 slow chargers with SAE J1772 connectors. The data is recorded from Nov. 2011 to Jan. 2015 containing 6800 charging events. In Case 2, the utilized data set is collected from on-street CPs in a local urban area of London, equipped with slow chargers with IEC62196 mode 1 connectors [36]. This data is provided by UK power networks and collected between Oct. 2012 and Feb. 2014, containing more than 14000 records. Two charging power levels 2.35 $\mathrm{kW}(10 \mathrm{~A})$ and $3.7 \mathrm{~kW}(16 \mathrm{~A})$ are considered in Case 2. The maximum attainable charging power not only depends on power rating of the CPs but also associates with the EVs' onboard chargers. Based on the simulation parameters indicated in Table I, the EVs' charging rate is calculated by multiplying $\eta_{c}$ by the minimum of $P_{c}$ and $P_{o}$. The EVs' discharging rate is calculated by multiplying $E_{c}$ and $\bar{v}$.

In order to validate the proposed Markov model and calibrate its parameters, it should be checked if the charging patterns randomly generated by the simulation process are close to the ones in available field data. The charging patterns include EV charging time, EV connection time and EV load profile. An attempt is made to obtain the best match between the simulation outputs and actual data by adjusting the simulation parameters through a search approach inspired by [45]. Prior to beginning the search process, an initial simulation run is performed using the predefined estimates of $p_{i}, q_{i}$ and $\epsilon_{t}$. These initial values are estimated based on the shape and statistical mean and variance of the pmf of EV charging time, the pmf of EV connection time and EV load profile obtained from historical data. The results of this initial run is checked for general resemblance to data. In every iteration of the search process, an incremental change to the simulation parameters is made in the neighboring area of the previously tuned parameters to find a better similarity between simulation outputs and data. If this change produces a better result, another incremental change is made in the next iteration, otherwise the direction of the change will be reversed. This iterative process continues until no further improvement can be found and the best acceptable accuracy has been reached. The goal is to gradually change and fine-tune the simulation parameters until the outputs have acceptable fit. This is a modified version of hill climbing technique. Two well-known goodness-of-fit tests are used as a tool for comparing the pmfs of the EV charging time and EV connection time derived from simulation with their equivalents derived from data, namely, two-sample Kolmogorov-Smirnov (KS) test and two-sample Anderson-Darling (AD) test [46]. These statistical procedures compare the two sets of data for a close match through a hypothesis test. In these tests, the null hypothesis that two empirical cumulative distribution values come from the same underlying distribution is accepted if the test statistic is smaller than the critical value of that test at a given significance level $\alpha$ or the p-value is greater than the significance level. The main advantage of the KS test is its sensitivity to the shape and scale of a distribution. Additionally, the AD test has two extra advantages over the KS test. First, it is much more sensitive towards differences at the tails of distributions. Second, the $\mathrm{AD}$ test is better capable of detecting very small differences, even between large sample sizes [46]. Utilization of these two statistical measures firmly confirm the resemblance between the simulation output distributions and the data.
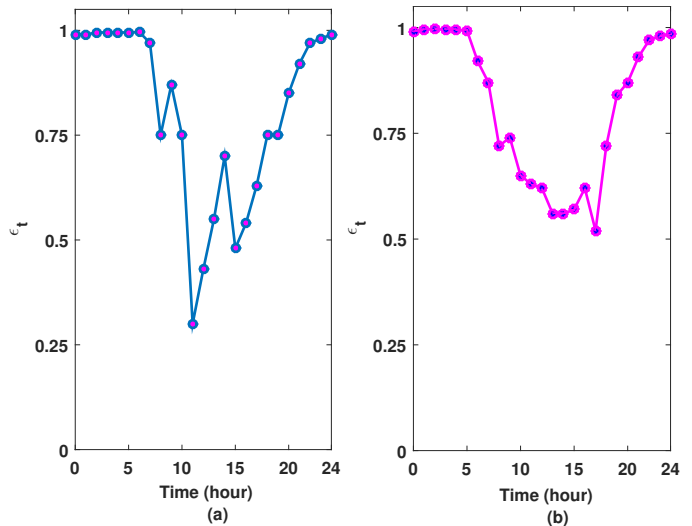

Fig. 5: The adjusted $\epsilon_{t}$ values in case studies (a) Case 1 (b) Case 2

In order to have a good estimate of the fit degree between the simulated EV load profile and the ones derived from the data, Mean Absolute Percentage Error (MAPE) is used as the error measure [47]. MAPE has been used to measure the accuracy of the proposed EV load modeling in [48]. MAPE is defined as

$$
\frac{1}{n} \sum_{i=1}^{n}\left|\left(\frac{y_{i}-x_{i}}{y_{i}}\right) \times 100\right|
$$

where $x_{i}$ is the simulated EV load value at time $\mathrm{i}$ and $y_{i}$ is the EV load value derived from the data at time $\mathrm{i}$. 


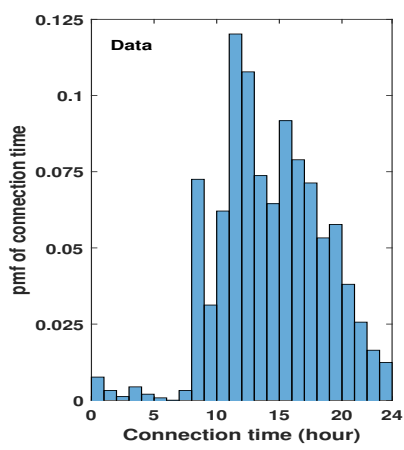

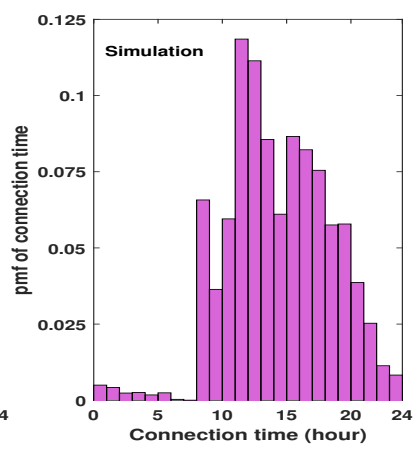

(a) Case 1
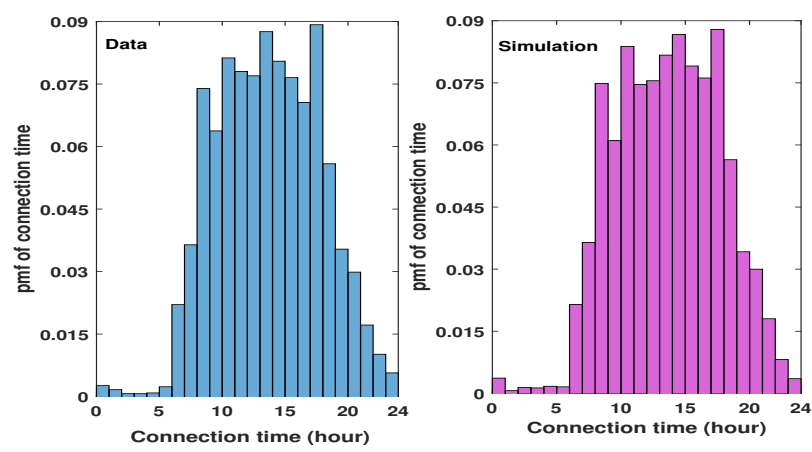

(b) Case 2

Fig. 6: pmf of EVs' connection time resulted from the field data and simulation
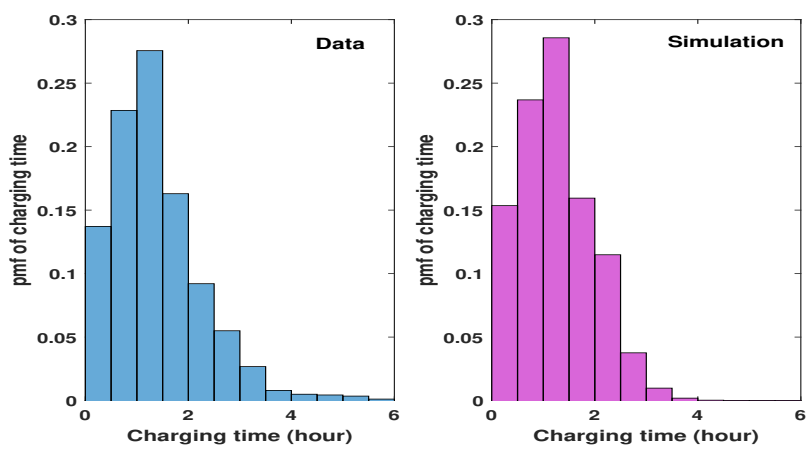

(a) Case 1
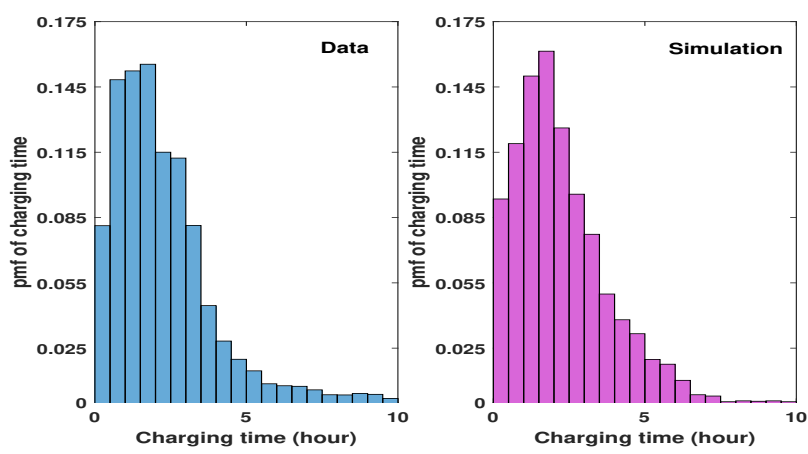

(b) Case 2

Fig. 7: pmf of EVs' charging time resulted from the field data and simulation
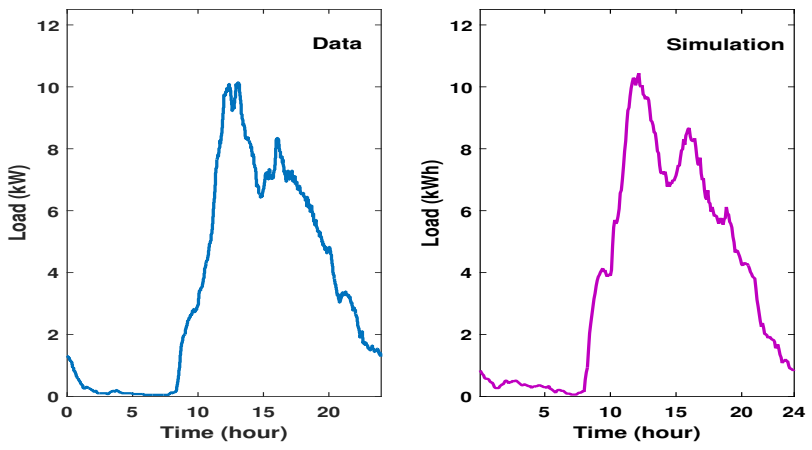

(a) Case 1
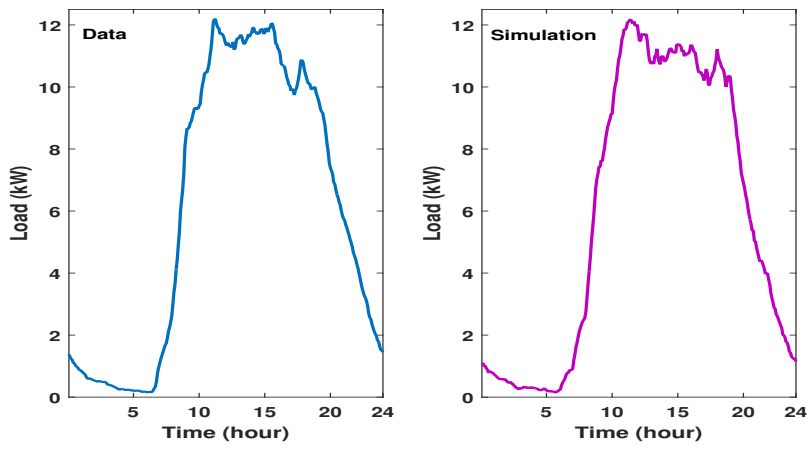

(b) Case 2

Fig. 8: EVs' load profile resulted from the field data and simulation

The tuned $p_{i}$ and $q_{i}$ parameters of the proposed Markov model for achieving the acceptable match between the simulated outputs and the data are obtained as described. These outputs, namely, the pmf of EVs' connection time, the pmf of EVs' charging time and EV load profile are shown in Figures 6 to 8 . The values of the obtained $\epsilon_{t}$ for every hour of a day in two case studies are shown in Fig. 5. In Case 1, the values of parameters $p_{i}$ and $q_{i}$ are tuned to $x_{p}=70, k_{p}=0.3, x_{q}=38$, $k_{q}=0.11$ by our iterative search method. In Case 2 , the community of EV drivers consists of two different behavioral groups due to the use of two types of CPs with different charging rates. $35 \%$ of EV drivers in the first group use the CPs with $3.7 \mathrm{~kW}$ charging rate and the others in the second group use slower $2.35 \mathrm{~kW}$ CPs. The values of $p_{i}$ and $q_{i}$ in first group are tuned to $x_{p}=80, k_{p}=0.16, x_{q}=35, k_{q}=0.13$ and the corresponding tuned values of parameters in second group are $x_{p}=75, k_{p}=0.21, x_{q}=22, k_{q}=0.08$. The drivers in the second group tend to charge their EVs more completely compared to the first group. They have enough time to leave their EVs at slower CPs. The drivers in the first group have a higher level of range anxiety.

The results of the goodness-of-fit tests over the outputs in Figures 6 and 7 are shown in Table II. The MAPE values obtained for the load profiles in Fig. 8 are 12.6\% and 11.74\% for cases 1 and 2, respectively. The MAPE is less than 13\% and the calibration accuracy is satisfactory. The results of these tests well justify the accuracy of our Markov model for driver's charging behavior. 
TABLE II: The results of goodness-of-fit tests for Figures 6 and $7(\alpha=5 \%$ and $\mathrm{AD}$ critical value $=2.492)$.

\begin{tabular}{ccccc}
\hline Test results & \multicolumn{2}{c}{$\begin{array}{c}\text { pmf of connection time } \\
\text { Fig. 6 }\end{array}$} & \multicolumn{2}{c}{$\begin{array}{c}\text { pmf of charging time } \\
\text { Fig. 7 }\end{array}$} \\
\hline & Case-1 & Case-2 & Case-1 & Case 2 \\
KS p-value & 0.5424 & 0.6195 & 0.2357 & 0.3982 \\
AD statistics & 1.0723 & 0.8162 & 1.8072 & 1.5248 \\
\hline
\end{tabular}

The $\epsilon_{t}$ setting and the shape of EV's connection time pmf confirm the behavioral characteristics of $\mathrm{EV}$ drivers in these simulated case studies. In Case 1, the drivers tend to connect the EVs to chargers mostly at 9 a.m., 12 noon and 4 p.m., as shown in Fig. 6a. In Case 2, the pmf of connection time fits well to a normal distribution and most of the connections occur between 9 a.m. and 19 p.m. with slightly more connections at 15 and 18 p.m., as depicted in Fig. 6b. These behaviors suggest that the charging process of EVs in these case studies mostly take place in urban areas, where the drivers leave their vehicles in parking lots for their daily activities, and naturally, parking lots will be empty from the late night until the early morning. The load profiles in Fig. 8 indicate that the number of daily charging events are very limited.

It is concluded that the simulation results acceptably justify the data analysis results in the two case studies. Therefore, the model parameters can be adjusted properly to predict the EV drivers' behavior in a small area. This achievement can help to predict the required capacity of CSs as well as to control the congestion in the stations and promote consumer satisfaction, thus, improving the profitability of the station owners. This idea will be assessed in Section V.

\section{Sensitivity Analysis}

In this section, a sensitivity analysis is performed to assess the influence of the proposed model parameters on the charging behavior by studying the pmf of EVs' charging times and connection times. $x_{p}$ (resp. $x_{q}$ ) is changed from 65 to 85 (resp. 20 to 40 ) and $k_{p}$ is increased from 0.13 to 0.63 . The pmf of charging times for 3 different values of these parameters are depicted in Fig. 9. It is observed that an adequate increase in $x_{p}$ (resp. decrease in $x_{q}$ ) leads to increasing the mean and variance and decreasing the skewness of the pmfs such that the shape of charging time pmf changes from nearly exponential to normal. Moreover, increasing the $k_{p}$ value leads to an increase in mean and variance of the charging time pmfs so that the shape of the pmf varies from exponential to normal distribution approximately. It can be shown that increasing the $k_{q}$ value has a similar effect on the charging time pmf with more sensitivity.

The results indicate that the charging time is distributed according to exponential distribution for some values of parameters, for example $x_{p}=65$ and/or $x_{q}=40$, which is similar to the assumptions made by [49]. While the charging time is distributed according to normal distribution for some other values of parameters similar to [32]-[34]. Although these assumptions seem to be contradictory, our result shows that they only differ because of the differences in the drivers' behaviors in different statistical communities. In a community whose EV drivers tend to connect when the initial battery level of charge is high and/or disconnect the EV with low battery level of charge, the charging time fits an exponential distribution very well. Decreased $x_{p}$ indicates the behavior of these drivers who do not stop at the parking lot for a long time and are unwilling to increase their vehicle's battery level of charge or they are more in a hurry to leave the parking lot. This is while, the behavior of EV drivers in an opposite way results in charging time with normal distribution. Decreased $x_{q}$ identifies the behavior of these kind of drivers who tend to connect when the battery level of charge reaches a smaller amount due to long-distance driving before entering the parking lot or having no access to a home charger. Furthermore, increased $k_{p}$ and $k_{q}$ represent the behavior of some drivers with a higher level of range anxiety in the latter community with a quick decision to connect or disconnect at a specific battery level of charge.

In our model, it is assumed that most of the drivers of a community have a few typical behaviors and the effect of minority on the overall system behavior can be ignored. In the first case study (i.e., Case 1), such a community with one typical behavior has been modeled. In the second case study (i.e., Case 2), we considered a community with two typical behavioral patterns. The results confirm that our model can be applied on a community where one or more typical behavioral patterns can be recognized. These behavioral groups are recognizable due to a variety of factors including the drivers' social habits, purpose of EV trips and type of EVs and CPs. To further emphasize this ability, we assessed the reported analysis on the data obtained from a community of electric taxi drivers operating in suburban areas of Beijing in [50]. This community belongs to one of the studied taxi platforms consisting of an EV fleet with $21 \mathrm{kWh}$ battery capacity and $0.15 \mathrm{kWh} / \mathrm{km}$ energy consumption coefficient. This platform is equipped with slow CPs that have $2.5 \mathrm{~kW}$ charging power.

The shape of EV charging time pmf of this community shows that it can be consisted of two distinct behavioral groups. In order to justify this idea, we simulated our model and adjusted its parameters in such a way that our resulted pmf of charging time nearly matches the outcome in [50], as depicted in Fig. 10. The adjusted model parameters in this simulation are as follows. Type 1 community members are $75 \%$ of the population and $x_{p}, k_{p}, x_{q}$, and $k_{q}$ are set as 85 , $0.6,25$, and 0.18 , respectively. Type 2 members are $25 \%$ and the same parameters, $x_{p}, k_{p}, x_{q}$, and $k_{q}$, are set as $60,0.47$, 20 , and 0.14 , respectively. This result reveals that the studied community of electric taxi drivers consists of two groups, as we guessed. $75 \%$ of the community members in group 1 have a higher level of range anxiety who tend to disconnect when the level of charge reaches near $85 \%$, while the EV drivers in group 2 have a lower level of range anxiety and disconnect earlier with $60 \%$ of charge level. Later we will show that the driven model can be helpful in predicting and managing congestion in CSs.

Till now, the effect of $p_{i}$ and $q_{i}$ on the charging time pmf have been studied. Now we assess the effect of $\epsilon_{t}$ on the pmf of connection time by performing two different scenarios named: ep-sample and ep-parking. The values of $\epsilon_{t}$ in these scenarios are assigned as shown in Fig. 11a and other parameters are 


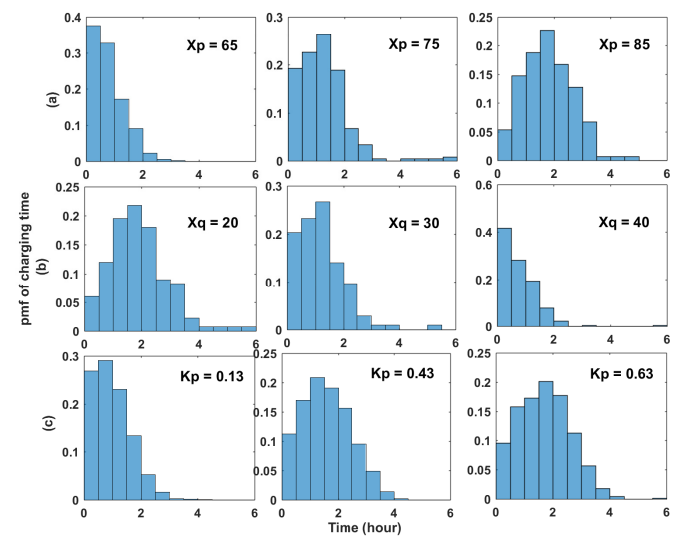

Fig. 9: PMF of charging time for 3 groups of parameters a) $x_{p}=65,75$, and 85 b) $x_{q}=20,30$, and 40 c) $k_{p}=0.13$, 0.43 , and 0.63

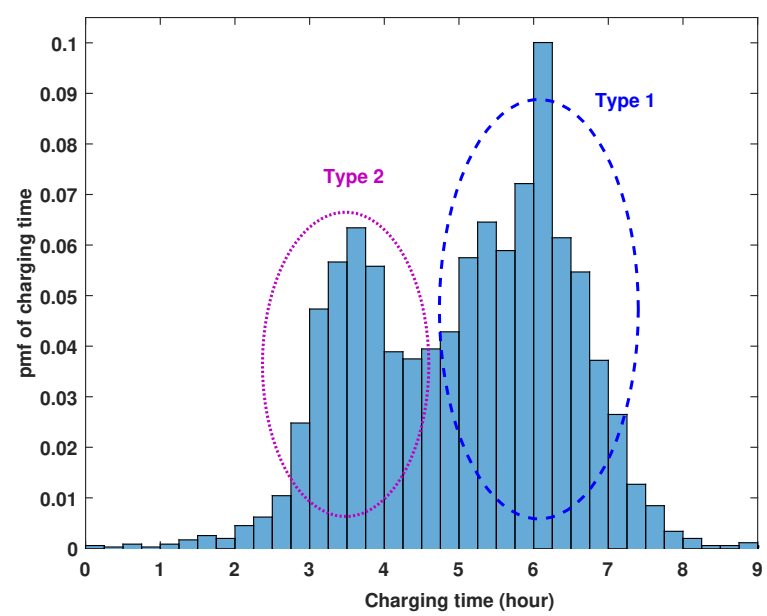

Fig. 10: The pmf of charging time in a complex community.

chosen from the first simulated case study in sec. III.

As it is mentioned earlier, $\epsilon_{t}$ describes the probability of parking the $\mathrm{EV}$ in time $\mathrm{t}$ when the $\mathrm{EV}$ is disconnected from the CP. It is observed from Fig. 11 that $\epsilon_{t}$ affects the drivers' behavior in deciding about the EV connection time in different places. The scenario ep-sample describes a relatively idle station which only gets busy at 13 p.m. and there is no connections at the early hours of the day. On the other hand, ep-parking presents a busy public parking lot which is crowded more at 7 a.m., 12 noon and 16 and 20 p.m.. The pmfs of connection time in these scenarios verifies the above assumptions, as depicted in Fig. 11b.

In summary, our analysis reveals that the different assumptions about the statistical characteristics of EV behavioralrelated parameters in the studied models are due to different EV drivers' behavior in different communities. In our presented model, we parameterize the charging behavior of EV drivers in such a way that all of their possible statistics can be produced. Furthermore, we suggest that our model will help researchers plan the future expansion of the CS and control the CS load profile by encouraging the drivers to change their EV charging behavior. In the next section, we will show how the CS congestion level can be managed and controlled by adjusting the behavioral parameters using the proposed
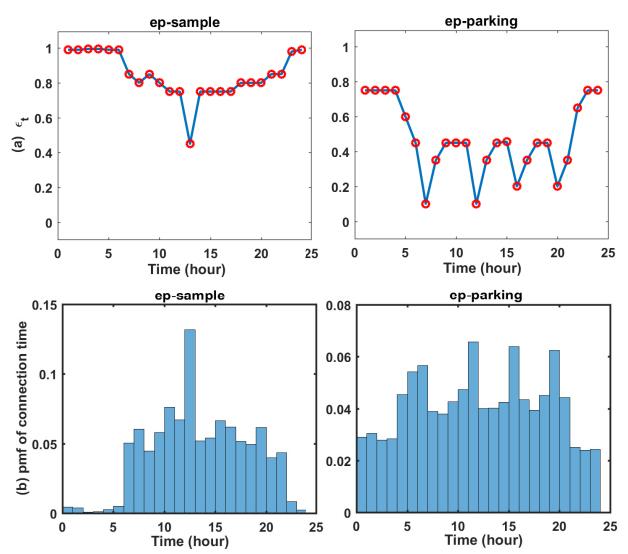

Fig. 11: a) $\epsilon_{t}$ for two test scenarios: ep-sample, ep-parking b) pmf of connection time for the test scenarios

model. To do so, we study the effect of the parameters on predicting the congestion event in a CS environment, when the number of EVs increases as it is foreseen.

\section{PREDICTING AND MANAGING CONGESTION IN CS DEVELOPMENT USING THE PROPOSED MODEL}

Congestion is a natural phenomenon when traffic is a matter of concern. An increase in the number of EVs in CSs increases the probability of service blocking, i.e., when an EV cannot connect to CPs because all CPs are in use. The blocking probability depends on the number of CPs and traffic intensity. In this case a driver has no choice but to leave the station and get the service later or in another CS. In the worst case, the $\mathrm{EV}$ battery is completely discharged before the driver gets the opportunity to find a free $\mathrm{CP}$ and he/she will become more discontent. We assume that the required power of CS is always and completely supplied by the power network for the sake of model simplicity. In Subsection V-A, the required number of CPs are predicted as the number of EVs increases in the future, for a predefined level of Quality of Service (QoS). In Subsection V-B, the effect of changing the behavioral parameters of the proposed model on the congestion status is assessed.

\section{A. Predicting the required station capacity subject to blocking probability constraint}

One of the important considerations in the development of the CSs and predicting their future capacity with the objective of limiting the blocking probability is studying the drivers' behavior in choosing the connection time of the day and charging durations. Specifically, introducing an appropriate level of QoS results in more consumer satisfaction and as a result more profit for station operators. Accordingly, two groups of scenarios are assessed to predict the number of CPs by increasing the number of EVs, while guaranteeing a certain level of QoS. Subsequently, the blocking probability is assessed with respect to the increased number of CPs by considering a fixed number of EVs in those scenarios. 
1) The effect of $p_{i}$ and $q_{i}$ on the predicted number of CPs: As indicated in Section IV, an increase in $x_{p}, k_{q}$ and $k_{p}$ and a decrease in $x_{q}$ lead to an increase in the average EV charging time. Therefore, drivers' behavior change is expected to have an effect on predicting the number of CPs. In order to study this effect, a group of scenarios are defined according to Table III. The $\epsilon_{t}$ vector is taken from Fig. 5a for all scenarios. Scenario 1 defines the behavior of the drivers in Case 1 based on real data, as described in Section III. In Scenario 2, EV drivers are used to partially charge their EVs with less duration of charge because they plan for a short travel distance or have a limited time. Conversely, drivers in Scenario 3 tend to completely charge their EVs. They connect the EV to the CP with a lower level of charge and disconnect with a higher level of charge. EV drivers in Scenario 4 (resp. Scenario 5) have a higher level of range anxiety compared to Scenario 2 (resp. Scenario 3) but their charging habits are similar. The blocking probabilities are computed by using the proposed model in the system simulation, and the behavioral parameters of the drivers determine the EV arrival times and charging durations.

The minimum number of required CPs in terms of the number of the existing EVs is determined while blocking probability of the fully depleted EVs is constrained to 0.05 , as shown in Fig. 12a. The obtained results indicate that only one CP is required in Scenario 4 even when the number of EVs is increased to 90 . This observation can be explained by considering the EV drivers behavior: 1) The drivers tend to connect to the CPs with higher battery level of charge to ensure the EV battery will not get out of charge in their next trip and continue charging until a lower level of charge is reached because they do not have enough time or do not want to spend more money and 2) The drivers have a higher level of range anxiety and decide to connect/disconnect to/from the CPs as soon as the EV battery reaches the predefined level of charge. Consequently, the EVs' charging time is decreased. This situation decreases the likelihood of EV blocking leading to complete battery depletion, despite the fact that the number of charging events increases. In contrast to the previous scenario, the largest number of CPs is required in Scenario 3 to meet the upper threshold requirement of the blocking probability.

TABLE III: $p_{i}$ and $q_{i}$ parameters for test scenarios

\begin{tabular}{ccccc}
\hline Scenario No. & $x_{p}$ & $k_{p}$ & $x_{q}$ & $k_{q}$ \\
\hline Scenario 1 & 70 & 0.3 & 38 & 0.11 \\
Scenario 2 & 60 & 0.3 & 45 & 0.11 \\
Scenario 3 & 90 & 0.3 & 20 & 0.11 \\
Scenario 4 & 60 & 0.45 & 45 & 0.2 \\
Scenario 5 & 90 & 0.45 & 20 & 0.2 \\
\hline
\end{tabular}

In the next simulation, we assess the effect of $p_{i}$ and $q_{i}$ on the congestion status in the CS, which leads to fully discharging the EVs. Therefore, the blocking probabilities of completely discharged EVs in previous scenarios are obtained with respect to the increased number of CPs from 2 to 30 by considering a fixed number of existing EVs equal to 50, as shown in Fig. 12b. Similar to the previous results, it is observed that there will never be any service blocking leading

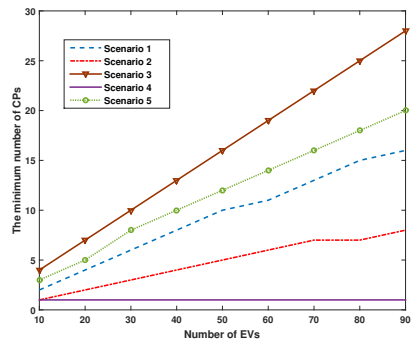

(a) Assessment 1 .

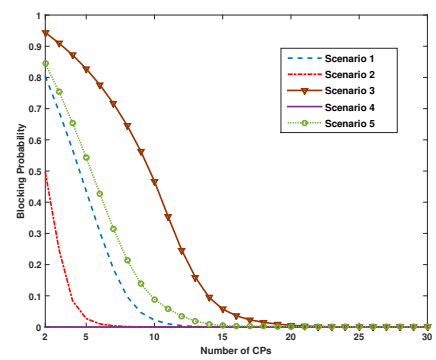

(b) Assessment 2.
Fig. 12: Assessment 1: Minimum number of required chargers in terms of EV count with congestion constraint: $P_{\text {block }}=$ 0.05 . Assessment 2 : The blocking probability of completely discharged EVs in terms of CPs count. The number of commuting EVs is 50.

to zero battery charge in Scenario 4, even when there is only one CP. This feature depends on the conservative behavior of EV drivers and shorter charging duration, as mentioned before. Every scenario with a higher $x_{q}$ and lower $x_{p}$ results in a lower number of CPs in order to limit the blocking probability to a certain level. The blocking probability in Scenario 4 is lower than its equivalent amount in scenario 2 . The reason is that the $\mathrm{EV}$ drivers have a higher level of range anxiety in this scenario in the sense that they want to connect the EVs when their EVs' battery level of charge reaches almost exactly $x_{q}$ level and disconnect it almost exactly at $x_{p}$ level. Therefore, the EV connection/disconnection takes place based on these amounts with less flexibility compared to the previous scenario. This result holds true in comparing Scenarios 5 and 3. Figure $12 \mathrm{~b}$ shows that the blocking probability of completely discharged EVs can be restricted to $20 \%$ when the minimum number of CPs in Scenario 2, Scenario 1, Scenario 5, and Scenario 3 are equal to $3,7,8$, and 12 respectively.

2) The effect of $\epsilon_{t}$ on the predicted number of CPs: In order to assess how a change in $\epsilon_{t}$ affects the congestion status, we simulate 4 scenarios, as shown in Fig. 13. In these scenarios, $\epsilon_{t}$ change frequency increases from scenario Busy Day Light (BDL) to Random $\epsilon(\mathrm{R} \epsilon)$ while its average is 0.4 . The minimum number of $\mathrm{CPs}$ required to constrain the blocking probability of the fully depleted EVs to the upper threshold level of 0.05 is shown in Fig. 14a.

According to these results, BDL requires the maximum number of CPs, since the CS is busy for a long continuous time and EV drivers are equally willing to connect to CPs with a high probability. In scenarios Busy Morning Afternoon (BMA) and $\mathrm{R} \epsilon$, the number of required CPs decreases while $\epsilon_{t}$ changes more frequently. It is noticeable that the effect of changes in $\epsilon_{t}$ on the congestion status depends on the battery capacity of EVs and the rate of battery depletion while driving. Therefore, BMA and $\mathrm{R} \epsilon$ act similarly since the rush hours in these scenarios are long enough to let some EVs get out of charge. On the other hand, scenario Constant $\epsilon(\mathrm{C} \epsilon)$ requires the minimum number of CPs because EV drivers are equally willing to visit the $\mathrm{CS}$ at all times with the probability of 0.4 , thus congestion is less likely to happen. Of course, the occurrence of such situation is unlikely in practice. It is concluded that the less the length of CS's busy periods and the 

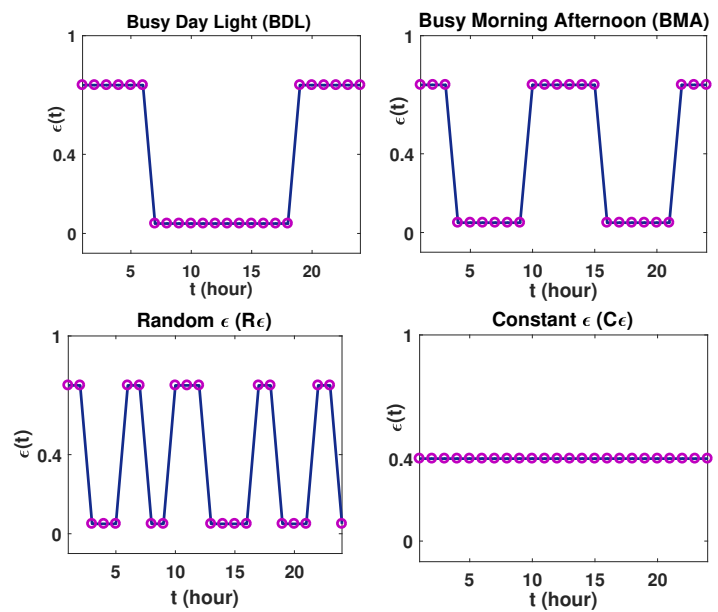

Fig. 13: $\epsilon_{t}$ values for test scenarios: Busy Day Light (BDL), Busy Morning Afternoon (BMA), Random $\epsilon(\mathrm{R} \epsilon)$ and Constant $\epsilon(\mathrm{C} \epsilon)$

more they are scattered over the day, the less number of CPs are required. Moreover, the assumption of EV arrivals based on a fixed rate leads to resource underprovisioning.

In the last simulation, the blocking probability is calculated in terms of the number of CPs with $50 \mathrm{EVs}$ in the area. The blocking probability for EVs getting fully discharged due to congestion is shown in Fig. 14b. As observed in this figure, BDL has the highest blocking probability among all, while $\mathrm{C} \epsilon$ has the lowest probability. By decreasing the blocking probability to less than 0.6 , the number of required CPs in BMA and $\mathrm{R} \epsilon$ decreases with respect to BDL. It is deduced that the more crowded BDL scenario increases the chance of EV service blocking, especially when the drivers tend to connect to CPs with a high probability in a long time period. Figure $14 \mathrm{~b}$ shows that the blocking probability of completely discharged EVs can be restricted to $20 \%$ when the minimum number of CPs in BDL to $\mathrm{C} \epsilon$ are equal to 26, 24, 22 and 16, respectively. After these points, an increase in the number of CPs by one unit decreases the blocking probability faster.

In the above scenarios, the blocking probability is defined as the probability of completely discharging the batteries because of congestion. This definition can be replaced by the probability of unsuccessful charge request, whether the battery is fully discharged or not. The new definition yields some changes in the results, e.g., the drivers with a higher level of range anxiety (larger $k_{p}$ and $k_{q}$ ) cause an increase in the blocking probability.

Accordingly, the major result is that the proposed model is suitable to apply in the capacity planning of CSs and control the blocking probability, when the number of EVs increases in the future. The CS operator can estimate the parameters of the model based on existing data to complete the model. Then, the required number of CPs will be predicted by the model simulation in order to guarantee a certain level of blocking probability. Furthermore, using the results of this model, by adjusting the $p_{i}$ and $q_{i}$ parameters appropriately the drivers' behavior can be manipulated and as a result the blocking probability in an existing CS can be controlled and its usage can be maximized. In the next section, we will study the effect (a) Assessment 1 .

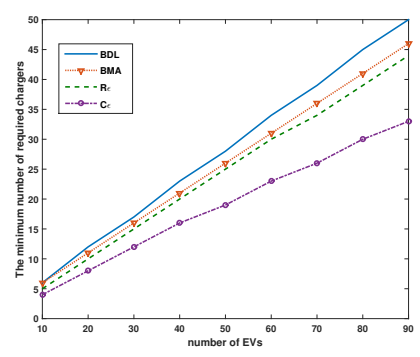

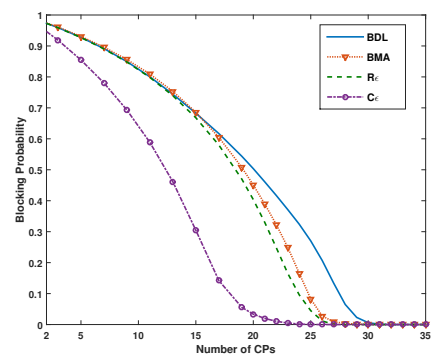

(b) Assessment 2.
Fig. 14: Assessment 1: Minimum number of required chargers in terms of EV count with congestion constraint: $P_{\text {block }}=$ 0.05. Assessment 2: The blocking probability of completely discharged EVs in terms of CPs count. The fixed number of commuting EVs is 50.

of changing $p_{i}$ and $q_{i}$ dynamically on the blocking probability in busy times in a typical CS and propose how to make use of the model to plan the CS.

\section{B. Congestion reduction by dynamically changing the model parameters}

Controlling the CS congestion is an important consideration in planning and developing a CS. In this regard, the EV drivers' behavior has a significant role. Therefore, it is suggested that smart pricing methods is used to encourage the drivers to change their behavior during the busy periods. Consequently, we run a simulation to study how dynamically changing the EV drivers' behavioral parameters in busy times in a CS will affect the blocking probability. We expect that these parameters can be adjusted by choosing a properly defined pricing scheme. In our simulation, the parameters of typical EV drivers in a typical CS with $30 \mathrm{CPs}$ are assumed to be: $x_{p}=80, k_{p}=0.45, x_{q}=30, k_{q}=0.20$ and $\epsilon_{t}$ is defined as shown in Fig. 5a. Based on these parameters, the busy period in the CS is known priorly which is from 11 am to $18 \mathrm{pm}$. The blocking probability is measured by increasing the number of EVs while the behavioral parameters are changed in the busy period. This is measured for each parameter individually, as shown in Fig. 15. The simulation results show that decreasing $x_{p}$ for the connected EVs and $x_{q}$ for disconnected EVs in busy periods will decrease the blocking probability but reducing $k_{p}$ and $k_{q}$ is less effective. The results obtained in Fig. 15a indicate that the effect of decreasing $x_{p}$ will reverse when the number of EVs increases above 85 because more EVs get the chance to connect in non-busy periods. This reverse effect is observed in Fig. $15 \mathrm{~d}$ when $k_{q}$ is reduced similarly. The blocking probability decreases by $15 \%$ at most when the number of EVs reaches to 65 and $x_{p}$ is reduced by $30 \%$. In Fig. 15b, decreasing $x_{q}$ becomes effective when the number of EVs goes beyond 60 . The blocking probability decreases by $5 \%$ when the number of EVs reaches to 85 and $x_{q}$ is reduced by $40 \%$ which indicates that $x_{q}$ reduction is less effective in comparison to $x_{p}$.

The outcome of the last simulation confirms that our proposed model can describe the charging behavior of EV drivers by the parameters $p_{i}$ and $q_{i}$ properly. As we expect, the driver 


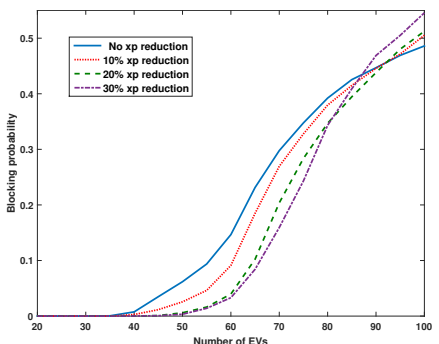

(a) $x_{p}$ effect.

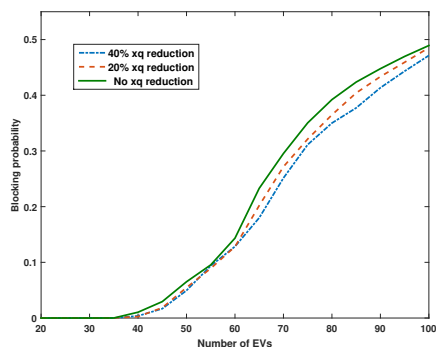

(b) $x_{q}$ effect.

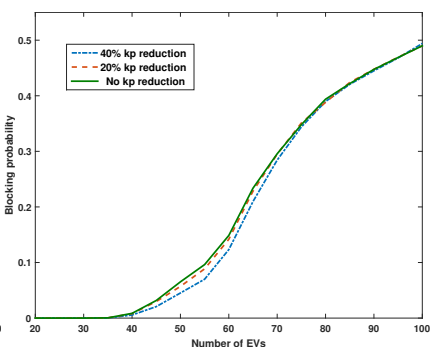

(c) $k_{p}$ effect.

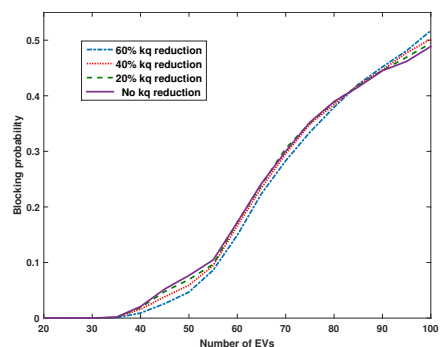

(d) $k_{q}$ effect.

Fig. 15: The effect of reducing behavioral parameters in the CS busy period.

can be encouraged to connect the EV later with less initial level of charge or disconnect the EV by less final level of charge in busy periods. Reducing $x_{q}$ for disconnected EVs and $x_{p}$ for connected EVs in busy periods shows the effect of this behavioral change on blocking probability. Consequently, the proposed model can be applied in planning and developing the CS in congestion status by changing the charging EV drivers' behavior properly. In this regard, developing peak power electricity pricing schemes is a key factor that incentivizes behavioral changes. In peak power electricity pricing, increasing the electricity price during busy periods will encourage the drivers to decrease $x_{p}, x_{q}, k_{p}$ and $k_{q}$ proportionally and the responsive drivers can benefit from cost reduction. In order to reach this goal, the proposed model can be used to analyze the CS to define the busy periods and the required changing amounts of behavioral parameters to reach the best level of blocking probability. It is noteworthy that over-increasing the electricity price in busy periods will have a reverse effect because EV drivers will decide to connect when the charging price is cheaper which increases the blocking probability again or they select a neighboring CS which decreases the CS profitability.

\section{DISCUSSION}

In the literature, several research efforts have been made in order to study the EVs charging demand, attempting to formulate the charging behaviors based on some generalized assumptions. Some of these assumptions fail to take into the account of real world EV charging patterns, while others are consistent with the analysis of real field data of EV charging events. For instance, authors in [18] assume that all vehicles leave home at the beginning of the day with their batteries fully charged. The parking duration, plug-in time and initial SoC are considered to follow a given normal distribution in [19], [23] and [41], respectively. Authors in [51] assume that the required charging energy for each parking event is proportional to the consumed energy during driving. However, a recent study on the historical data related to EV charging events shows that EV drivers appear considerably free to choose whether to charge their vehicles at home, workplaces or on-street parking lots [52]. Furthermore, they do not always start their day at full battery capacity. We have introduced a model to capture the stochastic nature of EV drivers' charging behavior without the above restrictive assumptions. In our case studies, we consider a constant charging power depending on the type of the $\mathrm{CP}$ which is one of the common assumptions in $\mathrm{EV}$ demand modeling [39]-[41]. This is compatible with constantcurrent constant-voltage (CCCV) battery charging behavior [52], especially when EVs are not allowed to be recharged once the SoC reaches $80 \%$. Moreover, we assume that the energy consumption during driving is proportional to the average EV velocity in the area of interest [13], [41]. While authors in [40] have further assessed the energy consumptions by taking into account the EV travel patterns and purpose of the trip, which is a more comprehensive assumption.

From the methodology point of view, we have proposed a Markov-based probabilistic model to emphasize the influence of EV drivers' charging behaviors on the statistical distributions of behavioral-related parameters and CS congestion management, and validated the model by real field EV data in various scenarios. Many studies have developed MCS based probabilistic models using transportation surveys or data collected from ICEVs. An agent based approach is employed in [15] to emulate the human aggregate behavior by considering predefined PDFs for some of decision variables. In [40] an EV driving pattern model based MCS is developed by defining three types of trips. Then EV transition probabilities are modeled using a Markov chain. Finally, expected charging profile is estimated using MCS based EV charging usage. A Markovbased analytical approach for modeling EV travel behavior and charging demand is presented in [41]. However, the EV travel behaviors are represented in several scenarios through MCS considering SoC and travel purposes. None of these models are validated using EV charging data. In other Markov-based models, the estimation of the transition state probabilities can be very time consuming due to considering the need for a high temporal resolution [13], [16]. The inhomogeneous Markov model introduced in [16] describes the driving pattern of one EV without considering the EV's characteristics and its driver's habits. The complication of this model prevents its applicability for studying the charging behavior of an EV fleet.

In author's opinion, modeling a typical EV driver's charging behavior in a statistical community of drivers is an applicable approach for studying the charging profile and aggregated behavior of the community. Our proposed model well introduces the driver's charging habits, especially by describing the dependency of charging decision to the battery level of charge and specifying the probability of parking the EV in 24 hours of the day which have direct influence on connection times and charging duration. Moreover $\epsilon_{t}$ can implicitly reflect the purpose of the Ev drivers' trip. Therefore, our model is flexible to describe the aggregated charging behavior of a 
statistical EV community while considering the type of CPs (i.e., slow or fast CPs), type of area under study and type of EVs (i.e., personal cars, company cars, taxis or buses). Furthermore, this model can be easily utilized to describe communities consisting of several groups of EV drivers with different behavioral characteristics. Another important aspect of the proposed model is that it provides an easy and applicable method for predicting required power capacity of the CS in terms of the number of CPs with respect to the increase of EVs in the future to prevent congestion in CPs.

\section{CONCLUSION AND FUTURE WORK}

In this paper, we proposed a new model to describe the charging behavior of an EV driver. The sensitivity analysis of the model indicated that the different assumptions about the statistical distributions of EVs' charging and connection times in the literature are because of the differences in the drivers' behaviors in different communities. This result confirms the ability of the model to differentiate the EV drivers' communities by reflecting their different behavioral characteristics. Moreover, we studied the effect of the model's behavioral parameters on the predicted number of CPs subject to the blocking probability constraint. The results showed that the required number of CPs decreases when EV drivers with a higher level of range anxiety tend to connect with a higher level of charge and disconnect faster with a lower level of charge and when the rush time periods are short and scattered over the day. Finally, we assessed the effect of changing our model parameters on the CS blocking probability in the busy periods. Reducing $x_{q}$ for disconnected EVs and $x_{p}$ for connected EVs in busy periods reduced the blocking probability which indicates the effect of changing the EV drivers' behavior on the CS congestion status. As a result, the behavioral parameters of the model can be changed by choosing a properly defined pricing scheme in order to control the CS congestion. Therefore, the proposed model facilitates the analysis of congestion status and predicting the required number of CPs to guarantee a required level of quality of service in CSs as the number of EVs increases in the future. This model can be applied to describe the real field data from any CS environment by adjusting the behavioral model parameters. Therefore, adopting this new model and studying the behavioral characteristics of different groups of drivers helps in planning the CSs to meet the increasing demand resulted from the increasing number of EVs.

In the future, a number of directions are considered worth further research effort. This work adopted a Markov-based probabilistic model to emphasize the influence of EV drivers' charging behavior on the CS congestion management due to its occupancy. The proposed model can be further extended and mathematically analyzed to consider the power constraints and congestion management of the CSs due to energy supply shortage when the penetration of EVs increases. Moreover, this model can be further utilized to investigate the effect of EV drivers' charging behavior on behavioral-related parameters covering a broad range of field statistical factors, e.g. purpose of EV trips, the service area of the CPs and usage type of EVs.
Furthermore, the proposed model can be utilized to study the effect of behavioral parameters on the congestion in a CS equipped with solar panels and battery storage units. Finally, the Bayesian inference and machine learning can be used to estimate the parameters of the proposed model in order to enhance the parameter estimation.

\section{REFERENCES}

[1] J. Y. Yong, V. K. Ramachandaramurthy, K. M. Tan, and N. Mithulananthan, "A review on the state-of-the-art technologies of electric vehicle, its impacts and prospects," Renewable and Sustainable Energy Reviews, vol. 49, pp. 365-385, 2015.

[2] C. M. Martinez, X. Hu, D. Cao, E. Velenis, B. Gao, and M. Wellers, "Energy management in plug-in hybrid electric vehicles: Recent progress and a connected vehicles perspective," IEEE Trans. Veh. Technol., vol. 66, no. 6, pp. 4534ph-4549, 2017.

[3] Y. Xue, J. You, X. Liang, and H. C. Liu, "Adopting strategic niche management to evaluate EV demonstration projects in china," Sustainability, vol. 8, no. 2, p. 142, 2016.

[4] H. C. Liu, X. Y. You, Y. X. Xue, and X. Luan, "Exploring critical factors influencing the diffusion of electric vehicles in china: A multistakeholder perspective," Research in Transportation Economics, vol. 66 , pp. 46-58, 2017.

[5] E. V. Initiative, Global EV outlook 2016, Beyond one million electric cars. International Energy Agency, Paris, 2016. [Online]. Available: https://www.iea.org/publications/freepublications/publication

[6] M. D. Galus, M. G. Vaya, T. Krause, and G. Andersson, "The role of electric vehicles in smart grids," Wiley Interdisciplinary Reviews: Energy and Environment, vol. 2, no. 4, pp. 384-400, 2013. [Online]. Available: http://dx.doi.org/10.1002/wene.56

[7] R. C. Green, L. Wang, and M. Alam, "The impact of plug-in hybrid electric vehicles on distribution networks: A review and outlook,' Renewable and sustainable energy reviews, vol. 15, no. 1, pp. 544-553, 2011.

[8] M. F. Shaaban, Y. M. Atwa, and E. F. El-Saadany, "PEVs modeling and impacts mitigation in distribution networks," IEEE Trans. Power Syst., vol. 28, no. 2, pp. 1122-1131, 2013.

[9] J. Van Roy, N. Leemput, S. De Breucker, F. Geth, P. Tant, and J. Driesen, "An availability analysis and energy consumption model for a flemish fleet of electric vehicles," Education, vol. 10, pp. 1-82, 2011.

[10] N. Machiels, N. Leemput, F. Geth, J. Van Roy, J. Büscher, and J. Driesen, "Design criteria for electric vehicle fast charge infrastructure based on flemish mobility behavior," IEEE Trans. Smart Grid, vol. 5, no. 1, pp. 320-327, 2014.

[11] E. Xydas, C. Marmaras, L. M. Cipcigan, N. Jenkins, S. Carroll, and M. Barker, "A data-driven approach for characterising the charging demand of electric vehicles: A uk case study," Applied Energy, vol. 162, pp. 763-771, 2016.

[12] F. Soares, J. P. Lopes, P. R. Almeida, C. Moreira, and L. Seca, "A stochastic model to simulate electric vehicles motion and quantify the energy required from the grid," in 17th Power Systems Computation Conf., 2011, pp. 22-26.

[13] P. Grahn, K. Alvehag, and L. Soder, "PHEV utilization model considering type-of-trip and recharging flexibility," IEEE Trans. Smart Grid, vol. 5, no. 1, pp. 139-148, 2014.

[14] H. Liang, B. J. Choi, W. Zhuang, and X. Shen, "Towards optimal energy store-carry-and-deliver for PHEVs via v2g system," in IEEE INFOCOM, 2012, pp. 1674-1682.

[15] K. S. Chaudhari, N. K. Kandasamy, A. Krishnan, A. Ukil, and H. B. Gooi, "Agent based aggregated behavior modelling for electric vehicle charging load," IEEE Trans. Ind. Informat., 2018.

[16] E. B. Iversen, J. K. Møller, J. M. Morales, and H. Madsen, "Inhomogeneous markov models for describing driving patterns," IEEE Trans. Smart Grid, vol. 8, no. 2, pp. 581-588, 2017.

[17] G. Souffran, L. Miègeville, and P. Guérin, "Simulation of real-world vehicle missions using a stochastic markov model for optimal powertrain sizing," IEEE Trans. Veh. Technol., vol. 61, no. 8, pp. 3454-3465, 2012.

[18] B. Da Lio, A. V. Guglielmi, and L. Badia, "Markov models for electric vehicles: the role of battery parameters and charging point frequency," in 20th IEEE Int. Workshop on Computer Aided Modelling and Design of Communication Links and Networks. IEEE, 2015, pp. 207-210.

[19] D. Tang and P. Wang, "Probabilistic modeling of nodal charging demand based on spatial-temporal dynamics of moving electric vehicles," IEEE Trans. Smart Grid, vol. 7, no. 2, pp. 627-636, 2016. 
[20] Q. Guo, S. Xin, H. Sun, Z. Li, and B. Zhang, "Rapid-charging navigation of electric vehicles based on real-time power systems and traffic data," IEEE Trans. Smart Grid, vol. 5, no. 4, pp. 1969-1979, 2014.

[21] J. Tan and L. Wang, "Real-time charging navigation of electric vehicles to fast charging stations: A hierarchical game approach," IEEE Trans. Smart Grid, vol. 8, no. 2, pp. 846-856, 2017.

[22] S. Deilami, A. S. Masoum, P. S. Moses, and M. A. Masoum, "Realtime coordination of plug-in electric vehicle charging in smart grids to minimize power losses and improve voltage profile," IEEE Trans. Smart Grid, vol. 2, no. 3, pp. 456-467, 2011.

[23] W. Su and M. Y. Chow, "Performance evaluation of an eda-based largescale plug-in hybrid electric vehicle charging algorithm," IEEE Trans. Smart Grid, vol. 3, no. 1, pp. 308-315, 2012.

[24] I. S. Bayram, G. Michailidis, M. Devetsikiotis, S. Bhattacharya, A. Chakrabortty, and F. Granelli, "Local energy storage sizing in plugin hybrid electric vehicle charging stations under blocking probability constraints," in IEEE Int. Conf. Smart Grid Commun. IEEE, 2011, pp. $78-83$.

[25] I. S. Bayram, G. Michailidis, M. Devetsikiotis, and F. Granelli, "Electric power allocation in a network of fast charging stations," IEEE J. Sel. Areas Commun., vol. 31, no. 7, pp. 1235-1246, 2013.

[26] I. S. Bayram, A. Tajer, M. Abdallah, and K. Qaraqe, "Capacity planning frameworks for electric vehicle charging stations with multiclass customers," IEEE Trans. Smart Grid, vol. 6, no. 4, pp. 1934-1943, 2015.

[27] C. Kong, R. Jovanovic, I. S. Bayram, and M. Devetsikiotis, "A hierarchical optimization model for a network of electric vehicle charging stations," Energies, vol. 10, no. 5, 2017. [Online]. Available: https://doi.org/10.3390/en10050675

[28] X. Dong, Y. Mu, H. Jia, J. Wu, and X. Yu, "Planning of fast EV charging stations on a round freeway," IEEE Trans. Sustain. Energy, vol. 7, no. 4, pp. 1452-1461, 2016.

[29] H. C. Liu, M. Yang, M. Zhou, and G. Tian, "An integrated multicriteria decision making approach to location planning of electric vehicle charging stations," IEEE Trans. Intell. Transp. Syst., no. 99, pp. 1-12, 2018.

[30] P. J. Tulpule, V. Marano, S. Yurkovich, and G. Rizzoni, "Economic and environmental impacts of a pv powered workplace parking garage charging station," Applied Energy, vol. 108, pp. 323-332, 2013.

[31] C. Lu, H. C. Liu, J. Tao, K. Rong, and Y. C. Hsieh, "A key stakeholderbased financial subsidy stimulation for chinese EV industrialization: A system dynamics simulation," Technological Forecasting and Social Change, vol. 118, pp. 1-14, 2017.

[32] T. Oda, M. Aziz, T. Mitani, Y. Watanabe, and T. Kashiwagi, "Mitigation of congestion related to quick charging of electric vehicles based on waiting time and cost-benefit analyses: A japanese case study," Sustainable Cities and Society, vol. 36, pp. 99-106, 2018.

[33] A. Mohamed, V. Salehi, T. Ma, and O. Mohammed, "Real-time energy management algorithm for plug-in hybrid electric vehicle charging parks involving sustainable energy," IEEE Trans. Sustain. Energy, vol. 5, no. 2, pp. 577-586, 2014.

[34] N. Liu and M. Cheng, "Effectiveness evaluation for a commercialized pv-assisted charging station," Sustainability, vol. 9, no. 2, p. 323, 2017.

[35] I. S. Bayram, V. Zamani, R. Hanna, and J. Kleissl, "On the evaluation of plug-in electric vehicle data of a campus charging network," in IEEE Int. Energy Conf. IEEE, 2016, pp. 1-6.

[36] U. P. Networks, Low Carbon London Electric Vehicle Load Profile. Greater London Authority, 2016. [Online]. Available: https://data.london.gov.uk

[37] X. Hu, C. Zou, C. Zhang, and Y. Li, "Technological developments in batteries: a survey of principal roles, types, and management needs," IEEE Power Energy Mag., vol. 15, no. 5, pp. 20-31, 2017.

[38] T. Zhang, X. Chen, Z. Yu, X. Zhu, and D. Shi, "A monte carlo simulation approach to evaluate service capacities of ev charging and battery swapping stations," IEEE Trans. Ind. Informat., vol. 14, no. 9, pp. 3914 3923, 2018.

[39] A. Ashtari, E. Bibeau, S. Shahidinejad, and T. Molinski, "PEV charging profile prediction and analysis based on vehicle usage data," IEEE Trans. Smart Grid, vol. 3, no. 1, pp. 341-350, 2012.

[40] A. Ul-Haq, C. Cecati, and E. El-Saadany, "Probabilistic modeling of electric vehicle charging pattern in a residential distribution network," Electric Power Systems Research, vol. 157, pp. 126-133, 2018.

[41] S. Sun, Q. Yang, and W. Yan, "A novel markov-based temporal-soc analysis for characterizing PEV charging demand," IEEE Trans. Ind. Informat., vol. 14, no. 1, pp. 156-166, 2018.

[42] Nissan Leaf. (2018) Range and charging. [Online] Available: https://www.nissan.co.uk/vehicles/new-vehicles/leaf/rangecharging.html
[43] M. Yilmaz and P. T. Krein, "Review of battery charger topologies, charging power levels, and infrastructure for plug-in electric and hybrid vehicles," IEEE Trans. Power Electron., vol. 28, no. 5, pp. 2151-2169, 2013.

[44] Scame electrical solutions for charging systems, "Ecomobility general catalogue 2015-2016", Tech. Rep. ZP00910-GB-3, 2016. [Online]. Available: www.scame.com

[45] J. Hourdakis, P. Michalopoulos, and J. Kottommannil, "Practical procedure for calibrating microscopic traffic simulation models," Transportation Research Record, no. 1852, pp. 130-139, 2003.

[46] S. Engmann and D. Cousineau, "Comparing distributions: the twosample anderson-darling test as an alternative to the kolmogorovsmirnoff test," Journal of Applied Quantitative Methods, vol. 6, no. 3, pp. 1-17, 2011.

[47] N. Daiheng, L. John, G. Angshuman, and W. Billy, "Systematic approach for validating traffic simulation models," Transportation Research Board, vol. 1876, pp. 20-31, 2004.

[48] S. Yang, M. Wu, X. Yao, and J. Jiang, "Load modeling and identification based on ant colony algorithms for EV charging stations," IEEE Trans. Power Syst., vol. 30, no. 4, pp. 1997-2003, 2015.

[49] M. Aunedi, M. Woolf, M. Bilton, and G. Strbac, "Impact and opportunities for wide-scale electric vehicle deployment," Report B1 for the "Low Carbon London LCNF project, Imperial College London, Tech. Rep., 2014. [Online]. Available: "http://www.ukpowernetworks.co.uk/innovation"

[50] Y. Zou, S. Wei, F. Sun, X. Hu, and Y. Shiao, "Large-scale deployment of electric taxis in beijing: A real-world analysis," Energy, vol. 100, pp. 25-39, 2016.

[51] Q. Huang, Q. S. Jia, Z. Qiu, X. Guan, and G. Deconinck, "Matching EV charging load with uncertain wind: A simulation-based policy improvement approach," IEEE Trans. Smart Grid, vol. 6, no. 3, pp. 1425-1433, 2015.

[52] Y. Xu, S. Çolak, E. C. Kara, S. J. Moura, and M. C. González, "Planning for electric vehicle needs by coupling charging profiles with urban mobility," Nature Energy, vol. 3, no. 6, pp. 484-493, 2018.

Zohreh Fotouhi received the B.Sc. degree in computer engineering and the M.Sc. degree in electrical engineering from Isfahan University of Technology, Iran. She is currently pursuing the Ph.D. degree in computer engineering at Isfahan University of Technology, Iran. Her research interests include smart grids, queuing theory, and computer networks.

Massoud Hashemi received the Ph.D. degree in electrical and computer engineering from the University of Toronto in 1998. From 1998 to 1999, he was a Post-Doctoral Fellow with the University of Toronto. Since 2003, he has been with the Isfahan University of Technology where he is currently an Associate Professor. His current research interests include software defined networks, information centric networking, and IoT.

Hamed Narimani received the B.Sc. and M.Sc. degrees from the Sharif University of Technology, Iran, in 2002 and 2004, respectively, and the M.Sc. and Ph.D. degrees from the Isfahan University of Technology, Iran, in 2009 and 2013, respectively, all in electrical engineering. He is currently an Assistant Professor with the Department of Electrical and Computer Engineering at Isfahan University of Technology. His main research interests are information theory and smart grid.

Islam Safak Bayram is a Scientist at Qatar Environment and Energy Research Institute both at Hamad Bin Khalifa University. He received the M.S. degree in Telecommunications from the University of Pittsburgh in 2010, and the Ph.D. degree in Computer Engineering from NC State University, in 2014. 